Bull. Austral. Math. Soc.

$46 \mathrm{G} 10,46 \mathrm{~B} 22$

VOL. 37 (1988) [323-332]

\title{
ON THE WEAK*-RADON NIKODYM PROPERTY
}

\author{
Elias SAAB
}

\begin{abstract}
We say that a certain property in a Banach space $E$ is stable by subspaces if every closed subspace of $E$ enjoys the same property. It is well known that the Radon-Nikodym property is stable by subspaces while the Weak Radon-Nikodym property is not. In his recent memoir, Talagrand investigated the stability of the Weak*Radon-Nikodym property which is a generalization of the Weak Radon-Nikodym property and showed that under Axiom L, the Weak*Radon-Nikodym property is stable by subspaces. It is still an open problem whether or not this result holds without this extra set theoretical hypothesis. In this paper we show that in a dual Banach space, the Weak*Radon-Nikodym property is stable by subspaces without assuming Axiom L. Other related results are discussed.
\end{abstract}

Let $E$ be a Banach space and $(\Omega, \Sigma, \mu)$ be a measure space. A function of $f: \Omega \rightarrow E$ is said to be scalarly measurable if for every $x^{*}$ in $E^{*}$, the function $x^{*} f$ is measurable. A scalarly measurable function is said to be Pettis integrable if for every $A$ in $\Sigma$ there exists an $x_{A}$ in $E$ such that

$$
x^{*}\left(x_{A}\right)=\int_{A} x^{*} f d \mu
$$

for every $x^{*}$ in $E^{*}$. If $f$ is Pettis integrable and strongly measurable, then $f$ is called Bochner integrable.

In case $E=F^{*}$ is the dual of a Banach space $F$, the function $f$ is said to be weak*-scalarly measurable if for every $x$ in $F$ the function $x f$ is measurable and if for each $A$ in $\Sigma$ there exists an $x_{A}^{*}$ in $E=F^{*}$ such that

$$
x_{A}^{*}(x)=\int_{A} x f d \mu
$$

for every $x$ in $F$, the function $f$ will be called weak*-integrable and $x_{A}^{*}$ will be denoted by $w^{*}-\int_{A} f d \mu$. The function $f$ is said to be weak*-scalarly bounded if for $x$ in $F$, the function $x f$ is bounded.

It is known $([8]$, p. 74$)$ that for any bounded linear operator

$$
T: L_{1}(\mu) \rightarrow E
$$

Received 25 June 1987

Copyright Clearance Centre, Inc. Serial-fee code: 0004-9729/88 \$A2.00+0.00. 
has a weak*-density, that is there exists a weak*-measurable, weak*-scalarly bounded function

$$
f: \Omega \rightarrow E
$$

so that

$$
\langle x, T g\rangle=\int g x f d \mu .
$$

It is also known $[8$, p. 36] that for any weak*-scalarly bounded function

$$
f: \Omega \rightarrow E
$$

there exists a bounded function

$$
g: \Omega \rightarrow E
$$

so that for every $x \in F, x f=x g \quad \mu$-almost everywhere. So every bounded linear operator

$$
T: L_{\mathbf{1}}(\mu) \rightarrow E=F^{*}
$$

has a bounded weak*density.

In the sequel, $E$ will stand for a Banach space that is not necessarily a dual space and $L^{1}$ for $L^{1}[0,1]$ endowed with Lebesgue measure $\lambda$.

The Banach space $E$ is said to have the $\mathrm{W}^{*} \mathrm{RNP}$ if every bounded linear operator $T: L^{1} \rightarrow E$ has a Pettis density valued in $E^{* *}$, it is said to have the B*RNP if every bounded linear operator $T: L^{1} \rightarrow E$ has a bounded weak* density valued in $E^{* *}$ that has the Bourgain property (see the definition below). Talagrand [8, p. 88] proved that under Axiom $L$, a Banach space $E$ has the $W^{*}$ RNP if and only if every subspace has the $W^{*}$ RNP. Here we show that a Banach space $E$ has the $B^{*} R N P$ if and only if every subspace of $E$ has the $\mathrm{B}^{*} \mathrm{RNP}$. We use this result to show that for dual Banach spaces the $W^{*} R N P$ is stable by subspaces without assuming Axiom $\mathrm{L}$.

All notations and notions used and not defined can be found in [1], [4], and [5].

DEFINITION 1: Let $(\Omega, \Sigma, \mu)$ be a measure space. A family $\psi$ of real-valued functions on $\Omega$ is said to have the Bourgain property if the following condition is satisfied: For each set $A$ of positive measure and for each $\alpha>0$, there is a finite collection $F$ of subsets of positive measure of $A$ such that for each function $f$ in $\psi$, the inequality

$$
0(f, B)=\sup f(B)-\inf f(B)<\alpha
$$

holds for some member $B$ of $F$.

It is known that if $\psi$ has the Bourgain property then

(1) the pointwise closure of $\psi$ satisfies the Bourgain property;

(2) each element in the pointwise closure of $\psi$ is measurable;

(3) each element in the pointwise closure of $\psi$ is the almost everywhere limit of a sequence from $\psi$. 
Definition 2: Let $(\Omega, \Sigma, \mu)$ be a measure space and let $E$ be a Banach space. A function $f: \Omega \rightarrow E$ is said to have the Bourgain property if the family $\left\{x^{*} f ; x^{*} \in\right.$ $\left.E^{*},\left\|x^{*}\right\| \leqslant 1\right\}$ has the Bourgain property.

REMARK: If $E=F^{*}$ is the dual of a Banach space $F$ then $f: \Omega \rightarrow E$ has the Bourgain property if and only if the family

$$
\{x f ; x \in F,\|x\| \leqslant 1\}
$$

has the Bourgain property.

Recall that ([4], p.521, Theorem 13) implies that if $f$ is bounded and has the Bourgain property then $f$ is Pettis-integrable. In [2] an example has been given of a Pettis-integrable function without the Bourgain property. The next proposition will allow us to give another example.

Proposition 3. Let $(\Omega, \Sigma, \mu)$ be a measure space and let $E$ be a Banach space. Let $f: \Omega \rightarrow E$ be bounded and has the Bourgain property then the set $\{m(A)=$ Pettis$\left.\int_{A} f d \mu ; A \in \Sigma\right\}$ is relatively compact in $E$.

Proof: First note that the set

$$
S=\left\{x^{*} f ; x^{*} \in E^{*} ;\left\|x^{*}\right\| \leqslant 1\right\}
$$

as a subset of $L^{1}(\Omega, \Sigma, \lambda)$ is compact, to see this let $x_{n}^{*} f$ be a sequence in $S$. Let $v$ be a cluster point of the sequence $\left(x_{n}^{*}\right)$ in $\left(B_{E^{*}}\right.$, weak*), therefore $v f$ is in the pointwise closure of $\left\{x_{n}^{*} f, n \geqslant 1\right\}$ which has the Bourgain property, hence there is a subsequence $x_{n_{k}}^{*} f$ converges to $v f$ in $L^{l}(\Omega, \Sigma, \lambda)$. A standard technique finishes the proof.

Fremlin and Talagrand [2] constructed a function $f:(\Omega, \Sigma, \mu) \rightarrow l^{\infty}$ that is Pettis integrable but such that the set

$$
\left\{\text { Pettis- } \int_{A} f d \mu ; A \in \Sigma\right\}
$$

is not relatively compact, hence this function does not have the Bourgain property.

Recall the following definitions:

Definition 4: A Banach space $E$ has the Radon Nikodym Property (RNP) [resp. the weak Radon Nikodym Property (WRNP)] if each bounded linear operator $T: L^{1} \rightarrow E$ has a Bochner density, (resp. a Pettis-density).

In [8], Talagrand showed that under axiom $(L)$ a Banach space $E$ has the $W^{*} \mathrm{RNP}$ if and only if every separable subspace of $E$ has the $\mathrm{W}^{*} \mathrm{RNP}$.

It is easy to see that the WRNP is not stable by subspaces. 
We will show that the $B^{*} R N P$ is always stable by subspaces (that is, without assuming Axiom L). To do that we need some preliminaries.

Let $\left(\pi_{n}\right)_{n \geqslant 1}$ be the sequence of the dyadic partition of $[0,1]$, and let $\Sigma_{n}$ denote the $\sigma$-algebra generated by $\pi_{n}$. Let $F$ be a Banach space and let $f:[0,1] \rightarrow F^{*}$ be a bounded function that is weak*-scalarly measurable. Consider the $F^{*}$-valued martingale $\left(f_{n}, \Sigma_{n}\right)$ where $f_{n}$ is defined by

$$
f_{n}=\sum_{A \in \pi_{n}} \frac{W^{*}-\int_{A} f d \lambda}{\lambda(E)} \chi_{A} .
$$

It was shown in [4] that if $f$ has the Bourgain property then the family

$$
\left\{\left\langle f_{n}, x\right\rangle ; n \in N, x \in F \quad\|x\| \leqslant 1\right\}
$$

has the Bourgain property. Using this we can now prove the following proposition. Before stating this proposition let $T: L^{1} \rightarrow E$ be a bounded linear operator and let

$$
g_{n}=\sum_{A \in \pi_{n}} \frac{T\left(\chi_{A}\right)}{\lambda(A)} \chi_{A}
$$

be its associated martingale.

Proposition 5. The operator $T: L^{1} \rightarrow E$ has a bounded weak* density in $E^{* *}$ that has the Bourgain property if and only if the set $H=\left\{\left\langle g_{n}, x^{*}\right\rangle ; x^{*} \in E^{*},\left\|x^{*}\right\| \leqslant 1\right.$; $n \geqslant 1\}$ has the Bourgain property.

Proof: We can suppose $\|T\|=1$. If $T$ has a bounded weak*-density $f:[0,1] \rightarrow$ $E^{* *}$ that has the Bourgain property then

$$
\begin{aligned}
g_{n} & =\sum_{A \in \pi_{n}} \frac{T\left(\chi_{A}\right)}{\lambda(A)} \chi_{A} \\
& =\sum_{A \in \pi_{n}} \frac{w *-\int_{A} f d \lambda}{\lambda(A)} \chi_{A} \\
& =f_{n} .
\end{aligned}
$$

Apply ([4], p.527, L.7 to L.-4) to see that $H=\left\{\left(g_{n}, x^{*}\right\rangle ; x^{*} \in E^{*},\left\|x^{*}\right\| \leqslant 1, n \geqslant 1\right\}$ has the Bourgain property.

Conversely, suppose that the set $H$ has the Bourgain property.

Let $g$ be a cluster point of the sequence $\left\{g_{n}\right\}$ in $B_{E^{* *}}^{[0,1]}$. Let $y^{*} \in E^{*},\|y *\| \leqslant 1$, then $y^{*} g$ belongs to the pointwise closure of the set

$$
\left\{\left\langle g_{n}, y^{*}\right\rangle ; n \geqslant 1\right\}
$$


which has the Bourgain property since it is a subset of $\mathrm{H}$. Hence $y^{*} g$ is measurable and there is a subsequence $\left(g_{n_{k}}\right)$ such that

$$
y^{*}(g(t))=\lim _{k} y^{*} g_{n_{k}}(t)
$$

$\lambda$-almost everywhere.

Let $h_{y^{*}}$ be a Radon-Nikodym derivative of $y^{*} T$ with respect to the Lebesgue measure $\lambda$. The sequence

$$
y^{*} g_{n_{k}}=\sum_{A \in \pi_{n_{k}}} \frac{y^{*} T\left(\chi_{A}\right)}{\lambda(A)} \chi_{A} .
$$

converges in $L^{1}$ to $h_{y^{*}}$, therefore for any Borel set $B$ in $[0,1]$

$$
\begin{aligned}
\lim _{k} \int_{B} y^{*} g_{n_{k}} d \lambda & =\int_{B} h_{y^{*}} d \lambda \\
& =y^{*}\left(T\left(\chi_{B}\right)\right)
\end{aligned}
$$

but by $\left({ }^{*}\right)$

$$
\lim _{k} \int_{B} y^{*} g_{n_{k}} d \lambda=\int_{B} y^{*} g d \lambda
$$

Hence $\int_{B} y^{*} g d \lambda=y^{*}\left(T\left(\chi_{B}\right)\right)$.

This shows that $g$ is a bounded weak ${ }^{*}$-derivative of $T$. To finish the proof, notice that the set

$$
M=\left\{x^{*} g ;\left\|x^{*}\right\| \leqslant 1\right\}
$$

is included in the pointwise closure of $H$. Hence $M$ has the Bourgain property. Consequently $T$ has a bounded weak* density $g$ valued in $E^{* *}$ that has the Bourgain property.

The next proposition will give another equivalent characterization of an operator $T: L^{1} \rightarrow E$ that has a bounded weak* density valued in $E^{* *}$ that has the Bourgain property.

A lifting of $L_{\infty}$ is a map $\rho$ from $L_{\infty}$ to the space measurable functions that is linear, multiplicative, positive such that $\rho(f)$ belongs to the class of $f$ for each $f \in L_{\infty}$.

A bounded subset $M$ of $L_{\infty}$ is said to satisfy "The Bourgain condition under lifting" [7] (if for (an equivalence class) of a set $A \subset[0,1], \lambda(A)>0$ and $\epsilon>0$, there is a partition $P=\left(A_{1}, \ldots, A_{n}\right)$ of $A$ into (equivalence classes of) subsets of $A, \lambda\left(A_{i}\right)>0$, such that for each $g \in M$, there is some $1 \leqslant i \leqslant n$ such that

$$
\text { ess } \sup g\left(A_{i}\right)-\operatorname{ess} \inf g\left(A_{i}\right)<\epsilon
$$


Proposition 6. An operator $T: L^{1} \rightarrow E$ has a bounded weak* density in $E^{* *}$ that has the Bourgain property if and only if $T^{*}\left(B_{E^{*}}\right)$ satisfies the Bourgain condition under lifting.

Proof: We can suppose $\|T\|=1$. If $T$ has a bounded weak*-density $f:[0.1] \rightarrow$ $E^{* *}$ that has the Bourgain property then

$$
T^{*}\left(B_{E^{*}}\right)=\left\{x^{*} f ;\left\|x^{*}\right\| \leqslant 1\right\}
$$

(Here we abuse the notation to really say that for each $g \in T^{*}\left(B_{E^{*}}\right)$ there is an $x^{*} \in B_{E^{*}}$ so that $g$ belongs to the class of $x^{*} f$.) Since $f$ has the Bourgain property, it follows immediately that $T^{*}\left(B_{E^{*}}\right)$ satisfy the Bourgain condition under lifting.

Conversely, suppose that $T^{*}\left(B_{E^{*}}\right)$ satisfies the Bourgain condition under lifting. Let $\rho$ be a lifting of $L_{\infty}$. Define

$$
g:[0,1] \rightarrow E^{* *}
$$

by $g\left\langle t, x^{*}\right\rangle=\rho\left(T^{*} x^{*}\right)(t)$. It is easy to see that $g(t)$ belong to $E^{* *}$ for any $t \in[0,1]$.

Since $T^{*}\left(B_{E^{*}}\right)$ satisfies the Bourgain condition under lifting, it follows that $g$ has the Bourgain property.

If $h \in L^{1}[0,1]$ then

$$
\left\langle T h, x^{*}\right\rangle=\left\langle h, T^{*} x^{*}\right\rangle=\int_{0}^{1} h T^{*} x^{*} d \lambda=\int_{0}^{1} h x^{*} g d \lambda
$$

so $g$ is bounded weak* density of $T$.

CoRollary 7. Let $E$ be a Banach space, then the following are equivalent:

(1) $E$ has the $B^{*} R N P$;

(2) Every subspace of $E$ has the $B^{*} R N P$;

(3) Every separable subspace of $E$ has the $B^{*} R N P$.

Proof: It is enough to show that $(1) \Rightarrow(2)$. Proving this boils down to using the Hahn-Banach Theorem coupled with either Proposition 5 or Proposition 6. Here we do it using Proposition 5. Let $F$ be a closed linear subspace of $E$ and let

$$
T: L^{1} \rightarrow F
$$

be a bounded linear operator and let $\left(g_{n}\right)_{n \geqslant 1}$ be its associated martingale. To see that $T$ has a bounded weak*-density $g$ valued in $F^{* *}$ it is enough to show that the set

$$
L_{F}=\left\{\left\langle g_{n}, y^{*}\right\rangle ; y^{*} \in F^{*},\left\|y^{*}\right\| \leqslant 1, n \geqslant 1\right\}
$$


has the Bourgain property and apply Proposition 5 .

But by Proposition 5 the set

$$
L_{E}=\left\{\left\langle g_{n}, y^{*}\right\rangle ; y^{*} \in E^{*},\left\|y^{*}\right\| \leqslant 1, n \geqslant 1\right\}
$$

has the Bourgain property.

Let $A$ be such that $\lambda(A)>0(\lambda$ is the Lebesgue measure on $[0,1])$ and let $\epsilon>0$, then there exists

$$
F_{1}, \ldots, F_{k} \subseteq A
$$

so that

$$
\lambda\left(F_{i}\right)>0
$$

and if

$$
\begin{gathered}
x^{*} \in E^{*},\left\|x^{*}\right\| \leqslant 1 \text { and } n \geqslant 1 \\
\min _{1 \leqslant i \leqslant k}\left(0\left(\left\langle g_{n}, x^{*}\right\rangle, F_{i}\right)\right) \leqslant \epsilon
\end{gathered}
$$

Let $\left\langle g_{n}, y^{*}\right\rangle \in L_{F}$. Extend $y^{*} \in F^{*}$ to an $x^{*} \in E^{*}$ such that $\left\|x^{*}\right\|=\left\|y^{*}\right\|$. Notice that $g_{n}$ is F-valued so

$$
\left\langle g_{n}, y^{*}\right\rangle=\left\langle g_{n}^{*}, x^{*}\right\rangle
$$

Hence

$$
\min _{1 \leqslant i \leqslant k}\left(0\left(\left\langle g_{n}, y^{*}\right\rangle, F_{i}\right)\right) \leqslant \epsilon
$$

Hence $L_{F}$ has the Bourgain property. This finishes the proof.

The following proposition is immediate:

Proposition 8. Let $E$ be a Banach space that is complemented in its bidual $E^{* *}$. If $E$ has the $W^{*} R N P$ then $E$ has the $W R N P$.

Proposition 9. Let $F=E^{*}$ be the dual of a Banach space $E$. Then the following statements are equivalent:

(1) The space $F$ has the WRNP.

(2) The space $F$ has the $W^{*} R N P$.

(3) The space $F$ has the $B \cdot^{*} R N P$.

Proof: $(2) \Rightarrow(1)$ is Proposition $8 .(3) \Rightarrow(2)$ is clear. To see that $(1) \Rightarrow(3)$, let $T: L^{1} \rightarrow E^{*}$ be a bounded linear operator. Let

$$
f:[0,1] \rightarrow E^{*}
$$


be a bounded weak*-density of $T$. For every $n \geqslant 1$ let

$$
\begin{aligned}
f_{n} & =\sum_{A \in \Pi_{n}} \frac{T\left(\chi_{A}\right)}{\lambda(A)} \chi_{A} \\
& =\sum_{A \in \Pi_{n}} \frac{w^{*}-\int_{A} f d \lambda}{\lambda(A)}
\end{aligned}
$$

be the martingale associated to $T$. If

$$
\left\{\left\langle f_{n}, x\right\rangle ; x \in E,\|x\| \leqslant 1, n \geqslant 1\right\}
$$

does not have the Bourgain property then $\left([4]\right.$, p.529, L.15) there exists a sequence $\left(x_{n}\right)$ in $E,\left\|x_{n}\right\| \leqslant 1$ so that the sequence $\left(\left\langle f, x_{n}\right\rangle\right)$ is equivalent to the $l_{1}$ basis in $L_{\infty}$. This implies that $\left(x_{n}\right)$ is equivalent to the $l_{1}$ basis in $E$, but this is impossible since $E^{*}$ has the $W R N P$.

Talagrand [8] showed that if a Banach space $F$ has the $W^{*} R N P$, then (under Axiom $L$ ) every subspace of $F$ has the $W^{*} R N P$.

The following Corollary shows that Talagrand's result is true without assuming Axiom $L$ if $F=E^{*}$.

Corollary 10. Let $F=E^{*}$ be the dual of a Banach space $E$. If $F$ has the $W^{*} R N P$, then every subspace of $F$ has the $W^{*} R N P$.

Proof: By Proposition 9, the space $F$ has the $B^{*} R N P$, hence by Corollary 7 every subspace of $F$ has the $B^{*} R N P$ and therefore every subspace of $F$ has the $W^{*} R N P$.

The above corollary allows us to give an example (without Axiom L) of a Banach space $E$ that has the $W^{*} R N P$ but not the $W R N P$.

Example 11: Let $X=(J T)^{*}$ be the dual of the James tree space. Since $X$ does not have the Radon-Nikodym Property, then $X$ has a separable subspace $E$ that does not have the Radon-Nikodym Property. This $E$ will have the $W^{*} R N P$ by Corollary 6 since $X$ has the $W^{*} R N P$.

The following proposition is a consequence of [3], Proposition 3, and Proposition 8.

Proposition 12. Let $E$ be a Banach lattice then the following are equivalent:

(1) The space $E$ has the RNP;

(2) The space $E$ has the WRNP;

(3) The space $E$ has the $W^{*} R N P$;

(4) The space $E$ has the $B^{*} R N P$. 
Let $E$ be a Banach space and $K$ be a weak*-compact subset of $E^{*}$.

Recall that $K$ is a Pettis set if the identity map

$$
\left(K, \text { weak }^{*}\right) \rightarrow(K,\|\|)
$$

is universally Pettis integrable $[4$, p.510, L.-9].

Definition 13: A weak*-compact subset $K$ of $E^{*}$ is a Bourgain set if for every Radon measure on $\left(K\right.$, weak $\left.^{*}\right)$ the identity map

$$
\left(K, \text { weak }^{*}\right) \rightarrow(K,\|\|)
$$

has the Bourgain property.

The following proposition shows that the notion of Pettis sets and Bourgain sets are the same, as suggested by Proposition 9.

Proposition 14. Let $K$ be a weak*-compact subset of $E^{*}$, then the following are equivalent:

(1) The set $K$ is a Bourgain set.

(2) The set $K$ is a Pettis set.

Proof: $(1) \Rightarrow(2)$ is evident. To see that $(2) \Rightarrow(1)$, let

$$
f:\left(K, \text { weak }^{*}\right) \rightarrow(K,\|\|)
$$

be the identity map. Let $\mu$ be a Radon measure on $K$. If $f$ does not have the Bourgain property, then by [4], there exists a sequence $\left(x_{n}\right)$ in the unit ball of $E$ so that the sequence $\left(\left\langle f, x_{n}\right\rangle\right)$ is equivalent to the usual $\ell_{1}$ basis in $L_{\infty}(K, \mu)$.

Let $f_{n}=\left(<f, x_{n}>\right)$. For each $n \geqslant 1, f_{n}: K \rightarrow \mathbf{R}$ defined by $f_{n}\left(x^{*}\right)=x^{*}\left(x_{n}\right)$.

Let $C(K)$ be the space of continuous function of $K$ considered a subset of $L_{\infty}(K, \mu)$. The sequence $\left(f_{n}\right)$ is included in $C^{\prime}(K)$ and is equivalent to the usual $\ell_{1}$ basis for the uniform norm on $C(K)$, hence by Rosenthal's theorem [5], the sequence $\left(f_{n}\right)$ does not have any subsequence that converges pointwise. On the other hand, the set $K$ is a Pettis set, so by $[6]$, there exists a sequence $\left(x_{n_{k}}\right)$ so that

$$
\lim _{k} x_{n_{k}}\left(x^{*}\right)
$$

exists for every $x^{*}$ in $K$. But this means that the sequence $\left(f_{n_{k}}\right)$ of $\left(f_{n}\right)$ converges pointwise. This contradiction finishes the proof.

REMARK: Walter Schachermayer (private communication) said that, using a recent result of Talagrand and Theorem 5.2 of [7], one can deduce that $B^{*} R N P$ is equivalent to strong regularity [7]. This will give a somewhat indirect proof of Corollary 7 . 


\section{REFERENCES}

[1] J. Diestel and J.J. Uhl Jr., Vector measures, Math Surveys 15 (Amer. Math. Soc., Providence, RI, 1977).

[2] D. Fremlin and M. Talagrand, 'A decomposition theorem for additive set functions and applications to Pettis integrals and erogodic means', Math. Z. 168 (1979), 117-142.

[3] N. Ghoussoub and E. Saab, 'On the weak Radon Nikodym property', Proc. Amer. Math. Soc. 81 (1981), 81-84.

[4] L. Riddle and E. Saab, 'On functions that are universally Pettis integrable', Mlinois J. Math. 20 (1985), 509-531.

[5] H. Rosenthal, 'A characterization of Banach spaces containing $\ell_{1}$ ', Proc. Nat. Acad. Sci., U.S.A. 71 (1974), 2411-2413.

[6] E. Saab, 'Some characterizations of weak Radon Nikodym sets', Proc. Amer. Math. Soc. 86 (1982), 307-311.

[7] W. Schachermayer, 'Operators from $L^{1}$ to Banach spaces and subsets of $L_{\infty}$ ', (pre-print).

[8] M. Talagrand, Pettis integral and measure theory 51, Memoirs of the Amer. Math Soc., 1984.

Department of Mathematics

University of Missouri

Columbia, MO 65211 